\title{
Selective growth of gold nanoparticles onto tellurium nanowires via a green chemical route $\dagger$
}

\author{
Zong-Hong Lin, Yang-Wei Lin, Kun-Hong Lee and Huan-Tsung Chang* \\ Received 30th January 2008, Accepted 19th March 2008 \\ First published as an Advance Article on the web 9th April 2008 \\ DOI: $10.1039 / b 801696 h$
}

In this paper, we describe a green chemical route for highly selective growth of Au tips onto Te nanowires (NWs). We carefully selected the $\mathrm{pH}$ values to vary the redox reaction potential between $\mathrm{Au}^{3+}$ and Te NWs, allowing control of nucleation and growth rates of $\mathrm{Au}$ nanoparticles (NPs). In the presence of $10 \mathrm{mM} \mathrm{CTAB}$, we obtained Au-Te and Au-Te-Au NWs at pH 4.0 and 5.0, respectively. Photovoltaic data revealed that the resistance of the Te NW-based thin films is controlled by the degree of deposition of Au NPs. We suspect that Au-Te and/or Au-Te-Au NWs hold great potential for use in the fabrication of electronic devices.

\section{Introduction}

The physical and chemical properties of nanomaterials are dependent on their composition ${ }^{1}$ and morphology. ${ }^{2}$ For example, anisotropic semiconductor nanostructures having metal tips on one or two of their termini have been prepared ${ }^{3}$ as potential materials for use in the fabrication of electronic devices. ${ }^{4}$ The presence of metal tips on nanomaterials is also desirable because it allows the anchoring of organic molecules or biomolecules $^{3 b, 5}$ and, hence, the sensing of various targets of interest. The symmetric growth of Au tips through the dodecylamine-mediated reduction of $\mathrm{AuCl}_{3}$ onto $\mathrm{CdSe}$ nanorods and tetrapods has been demonstrated in the presence of didodecyldimethylammonium bromide. ${ }^{3 b}$ At a Au salt-to-anisotropic nanostructure molar ratio of 15000 or more, one-ended growth of Au tips onto CdSe nanorods or tetrapods has been demonstrated to occur through Ostwald ripening. ${ }^{3 c}$ The same idea has been applied to the growth of $\mathrm{Au}$ tips onto $\mathrm{CdS}$ nanorods. ${ }^{3 d}$ Two-step approaches have also been demonstrated for the growth of metal tips onto nanomaterials. ${ }^{3 e}$ For instance, $\mathrm{PbSe}$ and CdTe nanocrystals have been grown selectively onto the tips of $\mathrm{CdSe}$ and $\mathrm{CdS}$ nanorods; those tips were subsequently oxidized by $\mathrm{Au}$ ions in the presence of surfactant to form Au tips on one end or both ends of the nanorods. Although these methods allow the preparation of metal tips on nanorods, it remains a challenge to prepare metal tips with high yield and purity on nanomaterials having lengths greater than $100 \mathrm{~nm}$. Moreover, most of the approaches described previously have been conducted in an organic phase at high temperature, i.e., against the principles of "green chemistry".

In this paper, we describe the development of a green-chemical route toward the selective growth of $\mathrm{Au}$ tips onto Te nanowires (NWs), using the redox reaction between $\mathrm{Au}^{3+}$ and Te NWs to deposit Au atoms in the form of Au nanoparticles (NPs) on the

Department of Chemistry, National Taiwan University, Taipei, Taiwan. E-mail: changht@ntu.edu.tw; Fax: +011-886-2-33661171; Tel: +011-886-2-33661171

$\dagger$ Electronic supplementary information (ESI) available: TEM images and UV-Vis absorption spectra of Au-Te NWs. See DOI: $10.1039 / \mathrm{b} 801696 \mathrm{~h}$ end(s) of Te NWs. In a previous study, we prepared Au-Te nanodumbbells, Au-Te nanopeapods, and Au pearl-necklace nanomaterials by carefully controlling the reaction time. ${ }^{6}$ Unfortunately, time control does not allow the growth of $\mathrm{Au}$ NPs in certain sizes or in desired positions on Te NWs. In our present study, careful control over the $\mathrm{pH}$ allowed us to vary the redox reaction potential between $\mathrm{Au}^{3+}$ and the Te NWs and, thus, control the rate of nucleation and growth of the Au NPs. With this approach we prepared $\mathrm{Au}-\mathrm{Te}$ (one Au tip) and Au-Te$\mathrm{Au}$ (two Au tips) NWs in high yield and purity. We found that controlling the deposition of $\mathrm{Au}$ NPs onto Te NWs provided nanomaterials with a range of conductivities.

\section{Experimental}

\section{Materials}

Sodium dodecyl sulfate (SDS, 99\%) and hexadecyltrimethylammonium bromide (CTAB, 99\%) were obtained from Acros. Sodium tetrachloroaurate dihydrate $(99 \%)$ was obtained from Aldrich. Tellurium dioxide $(99.9 \%)$ and hydrazine monohydrate $(80 \%)$ were purchased from Showa. Deionized water from a Milli-Q ultrapure $(18.2 \mathrm{M} \Omega \mathrm{cm})$ system was used in this study.

\section{Synthesis of Te NWs}

Two differently sized Te NWs (lengths/diameters: 420/30 and $950 / 40 \mathrm{~nm}$ ) were prepared through the reduction of tellurium dioxide with hydrazine and the deposition of Te atoms (either reduced from telluride ions or dissolved from amorphous tellurium NPs) onto trigonal Te nanocrystallites. ${ }^{7}$ Typically, hydrazine $(50 \mathrm{~mL})$ was added slowly to a beaker containing tellurium dioxide $(0.16 \mathrm{~g})$ at room temperature under constant magnetic stirring. The solution changed from colorless to blue after $40 \mathrm{~min}$, indicating the formation of Te NWs. Reaction times of 60 and $120 \mathrm{~min}$ provided the 420 and $950 \mathrm{~nm} \mathrm{Te}$ NWs, respectively. The reactions were terminated by diluting the mixtures 10 -fold with $10 \mathrm{mM}$ SDS. Prior to use in the preparation of Au-Te and Au-Te-Au NWs, the as-prepared Te NWs were subjected to a centrifugation/wash cycle to remove most of the matrix, including SDS and hydrazine. 


\section{Selective growth of Au NPs}

Through a redox reaction between $\mathrm{Au}^{3+}$ ions and the Te NWs, $\mathrm{Au}$ atoms were deposited to form Au NPs on the end(s) of Te $\mathrm{NWs}$, while $\mathrm{TeO}_{3}{ }^{2-}$ ions were formed and released into the bulk solution. ${ }^{6,8}$ In a typical synthesis, the Te NWs were redispersed in $10 \mathrm{mM} \mathrm{CTAB}$; after $30 \mathrm{~min}, 0.1 \mathrm{M} \mathrm{HCl}$ was added to the mixture to adjust the $\mathrm{pH}$ to a desired level ( $\mathrm{pH} 4.0$ for one-ended growth; pH 5.0 for double-ended growth). After $1 \mathrm{~min}, 10 \mathrm{mM}$ aqueous sodium tetrachloroaurate $(80 \mu \mathrm{L})$ was added to each solution and the mixture maintained at room temperature for $1 \mathrm{~h}$ to complete the reaction.

\section{Photovoltaic measurements of Te NW-, Au-Te NW-, and Au-Te- Au NW-based thin films}

Prior to measurements, the NWs $(100 \mathrm{~mL})$ were centrifuged at $6000 \mathrm{rpm}$ for $10 \mathrm{~min}$ and the pellets redispersed in deionized water $(2 \mathrm{~mL})$. The solutions were then added dropwise onto fluorinedoped tin oxide (FTO) glass and dried at room temperature. Counter electrodes were prepared by dropping Pt NPs (synthesized using the polyol method $)^{9}$ onto FTO glass and then sintering at $450{ }^{\circ} \mathrm{C}$ for $30 \mathrm{~min}$. Each FTO glass and a counter electrode were sealed with a spacer to fabricate a sandwiched device. Photovoltaic measurements were conducted using an Oriel 450 W Xe Arc lamp, a high-pass filter $(725 \mathrm{~nm})$, and a Keithley 2400 source meter.

\section{Characterization}

JEOL-1200EX II and FEI Tecnai-G2-F20 TEMs were used to measure the sizes and shapes of the prepared nanomaterials. EDX analyses were used to confirm the compositions of the prepared nanomaterials. XRD measurements were performed using a PANalytical X'Pert PRO diffractometer and $\mathrm{Cu} \mathrm{K} \alpha$ radiation $(\lambda=0.15418 \mathrm{~nm})$; the samples were prepared on glass substrates. UV-Vis absorption spectra of the colloids were recorded using a double-beam UV-Vis spectrophotometer (Cintra 10e, GBC).

\section{Results and discussion}

Fig. 1 displays the time evolution of the one-ended growth of $\mathrm{Au}$ NPs onto Te NWs having lengths and diameters of $420 \pm 60$ and $30 \pm 4 \mathrm{~nm}$, respectively. After redox reactions between $\mathrm{Au}^{3+}$ and Te NWs at pH 4.0 for 10, 30, and 60 min, we obtained Au NPs having diameters of $20 \pm 2 \mathrm{~nm}$ (Fig. 1B), $50 \pm 4 \mathrm{~nm}$ (Fig. 1C), and $70 \pm 5 \mathrm{~nm}$ (Fig. 1D), respectively, on only one end of the Te NWs. The final $\mathrm{pH}$ range of the solutions was 3.8-3.9. TEM images obtained over time suggest that the growth mechanism in our case differed from those reported previously, ${ }^{3 b-e}$ i.e., initial growth of Au NPs on both ends of the nanorod with one Au tip growing to greater size and becoming more stable while the other dissolved through Oswald ripening. Recently, Au-Pd nanostructures have been synthesized through a ripening process. ${ }^{10}$

When we used energy dispersive X-ray (EDX) analysis to characterize both ends of the Te-Au NWs presented in Fig. 1D, we observed (Fig. 2A) an Au signal on only one of the termini. The HRTEM images display the lattice spacings of Au (111) planes in the Au tip and Te (001), (010), and (110) planes in the Te part. Powder X-ray diffraction (XRD) measurements

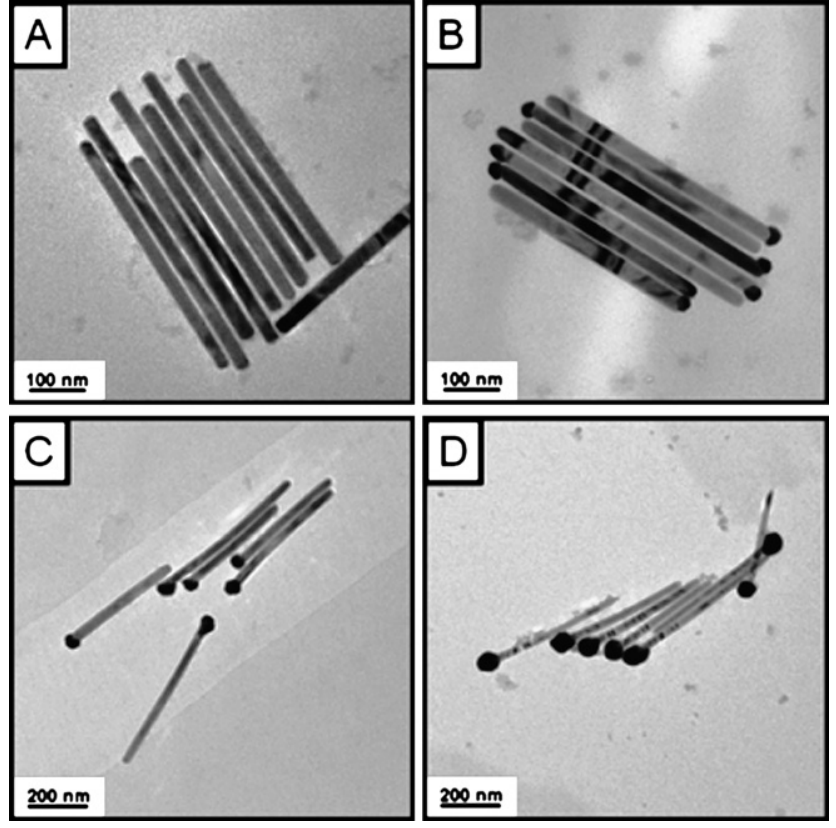

Fig. 1 TEM images of Au-Te NWs obtained from Te NWs (length: $420 \pm 60 \mathrm{~nm}$; diameter: $30 \pm 4 \mathrm{~nm}$ ) after reaction times of (A) 0 , (B) 10 , (C) 30, and (D) $60 \mathrm{~min}$.

revealed the presence of $\mathrm{Au}$ NPs in the Au-Te NWs. We detected the (102), (110), (111), (003), (200), (201), (112), (202), (113), (210), (211), (104), and (212) crystal facets of trigonal Te in XRD analysis of the pure Te NWs. The (003), (200), (112), (202), (113), (210), (211), (104), and (212) crystal facets were undetectable and the characteristic crystal facets for Au at (111), (200), (220), and (311) become apparent in the XRD analysis (Fig. 2B) of the Au-Te NWs possessing a single $70 \mathrm{~nm} \mathrm{Au} \mathrm{NP} \mathrm{(Fig.} \mathrm{1D).}$

Next, we demonstrated the preparation of Au-Te NWs using Te NWs having lengths and diameters of $950 \pm 160$ and $40 \pm 5$ $\mathrm{nm}$, respectively, at $\mathrm{pH}$ 4.0. The TEM image in Fig. S1A (ESI $\dagger$ ) reveals that we had prepared high-purity (ca. 100\%) Au-Te NWs. Upon increasing the $\mathrm{Au}^{3+}$ concentration from 80 to $100 \mu \mathrm{M}$, the diameters of the Au NPs on the Au-Te NWs increased from 50 $( \pm 6)$ to $70( \pm 10) \mathrm{nm}($ ESI $†$ Fig. S1A and S1B). Because of the presence of these large $\mathrm{Au}$ NPs on the Au-Te NWs, their characteristic surface plasmon resonance (SPR) absorption at $560 \mathrm{~nm}$ become observable (ESI $\uparrow$ Fig. S1C). The 420 and $950 \mathrm{~nm}$ Au-Te NWs absorb light over the spectral range from 200 to $1200 \mathrm{~nm}$, with absorption maxima at 650 and $720 \mathrm{~nm}$, respectively. We note that the lengths of these two Au-Te NWs were similar to those of their respective original Te NWs. The solutions of the Au-Te NWs were both purple, quite different from the blue color of their corresponding Te NWs. The purple hue resulted from the mixing of the blue color of the Te NWs and the red color of the Au NPs.

Upon increasing the $\mathrm{pH}$ of the reaction mixture to 5.0, we prepared $\mathrm{Au}-\mathrm{Te}-\mathrm{Au} \mathrm{NWs}$ having $\mathrm{Au} \mathrm{NP}$ diameters of $40 \pm 10$ $\mathrm{nm}$ (Fig. 3A). The final solution $\mathrm{pH}$ value was 3.9. From an analysis of $100 \mathrm{Au}-\mathrm{Te}-\mathrm{Au} \mathrm{NWs}$, we found that the sizes of the $\mathrm{Au}$ NPs at the two ends were almost identical (to within $\pm 2 \mathrm{~nm}$ ); such homogeneity suggests that the Au NPs grew simultaneously. ${ }^{3 d}$ The size distribution of the Au NPs on the ends of 

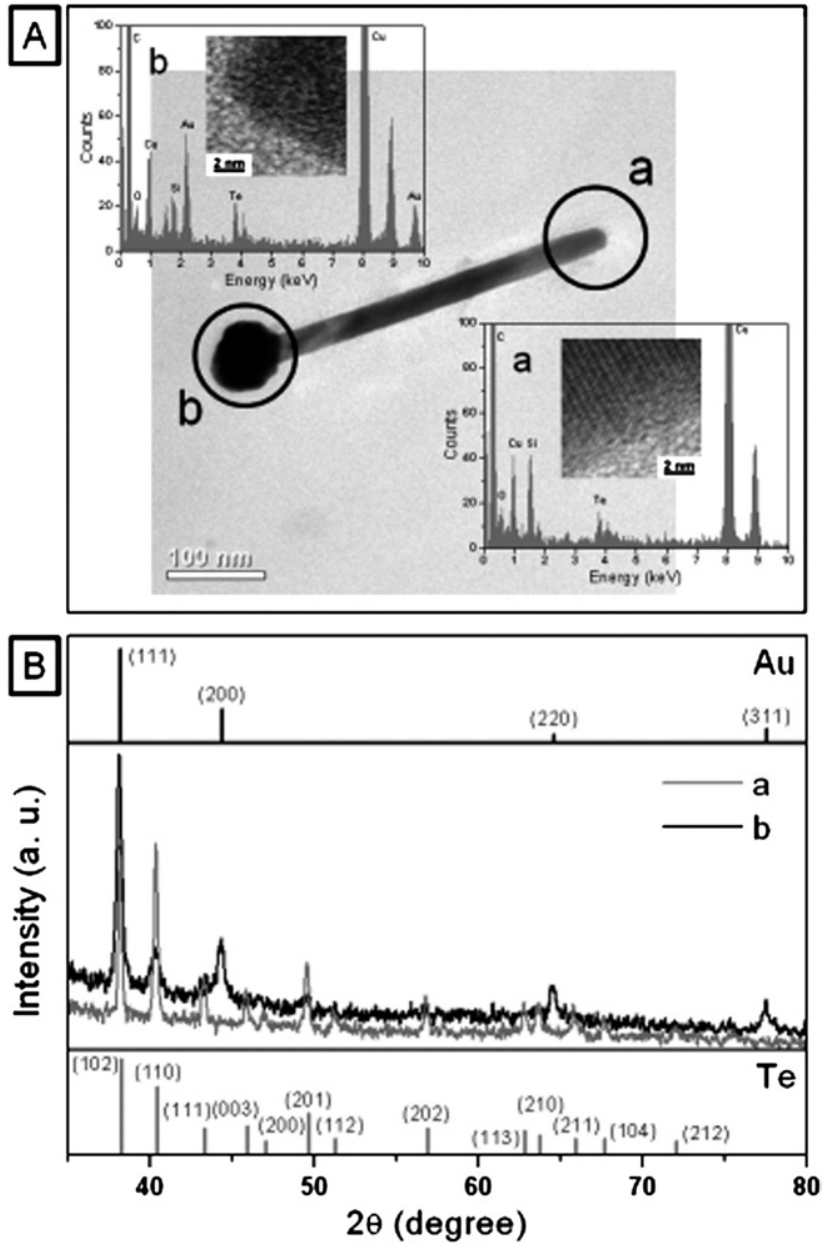

Fig. 2 (A) EDX analyses and HRTEM images of the ends (a) without and (b) with a Au NP of one of the Au-Te NWs presented in Fig. 1D. The characteristic peaks for elemental $\mathrm{Au}$ are absent in spectrum (a). The HRTEM image of Au tip shows interplanar spacings of $0.24 \mathrm{~nm}$, which corresponds to the $\mathrm{Au}$ (111) lattice planes. The interplanar spacings of $0.59,0.39$, and $0.22 \mathrm{~nm}$ correspond to the (001), (010), and (110) lattice planes of trigonal Te. (B) Powder XRD patterns of the initial Te NWs and the Au-Te NWs presented in Fig. 1D. The upper and lower spectra represent the XRD patterns of bulk $\mathrm{Au}$ and Te, respectively. The diffraction peaks in spectrum (b) were indexed to a combination of the Te and Au peaks.

Au-Te-Au NWs (RSD $=25 \% ; n=100)$ was slightly broader than that of the Au-Te NWs (RSD $=12 \% ; n=100)$. Further increasing the reaction mixture's $\mathrm{pH}$ to 6.0 resulted in smaller $\mathrm{Au}$ NPs (average diameter: $30 \pm 7 \mathrm{~nm}$ ) on the ends of the Te NWs (Fig. 3B). When we performed the procedure in solutions at values of $\mathrm{pH}$ ranging from 7.0 to 9.0, the Au atoms deposited at various positions along the Te NWs to form $\mathrm{Au}-\mathrm{Te}$ nanopeapods $(\mathrm{ESI} \dagger$ Fig. S2). These results indicate that the $\mathrm{pH}$ was an important factor that controlled the deposition of the Au NPs on the Te NWs. Upon increasing the $\mathrm{pH}$, the rate (potential) of the redox reaction between $\mathrm{Au}^{3+}$ and Te NWs increases:

$$
4 \mathrm{Au}^{3+}+3 \mathrm{Te}+6 \mathrm{H}_{2} \mathrm{O} \rightarrow 4 \mathrm{Au}+3 \mathrm{TeO}_{2}+12 \mathrm{H}^{+}
$$

As a result, the rate of formation of Au seeds increases. At high values of $\mathrm{pH}$, smaller-sized $\mathrm{Au}$ NPs deposited on the Te NWs
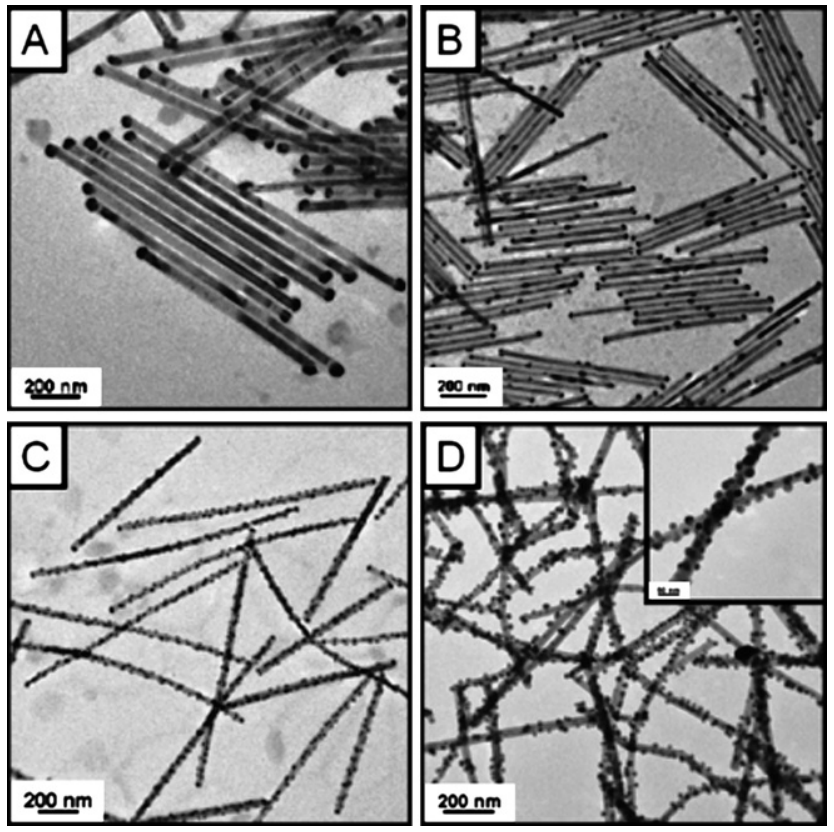

Fig. 3 TEM images of Au-Te nanomaterials prepared at $\mathrm{pH}$ (A) 5.0 and (B) 6.0 in $10 \mathrm{mM} \mathrm{CTAB}$ and at $\mathrm{pH} 4.0$ in (C) 0.5 and (D) $0.1 \mathrm{mM}$ CTAB.

without positional control. In contrast, larger Au NPs deposited selectively on the end(s) of the Te NWs at low pH. Next, we investigated the role played by CTAB during the preparation of the Au-Te NWs at $\mathrm{pH}$ 4.0. We suspected that CTAB bilayers protected the longitudinal edges of Te NWs,${ }^{11}$ so that the Au ions had better access to the end(s) ${ }^{12}$ where, consequently, the Au NPs deposited favorably. To test this hypothesis, we prepared Au-Te nanomaterials at various concentrations of CTAB $(0.1-10 \mathrm{mM})$. At concentrations less than $1 \mathrm{mM}$, Au atoms were not deposited selectively onto the end(s), as the TEM images in Fig. 3C and D reveal. This finding supports our notion that CTAB adsorbs preferentially onto the longitudinal edges of Te NWs. We point out that a cation exchange process was conducted to prepare $\mathrm{CdS}-\mathrm{Ag}_{2} \mathrm{~S}$ nanorods. ${ }^{13}$

Te is a semiconductor having a band gap of $0.3 \mathrm{eV}$, and which can be subjected to photostimulated electron transfer under irradiation in the near-IR region. A Te NW-based thin film provided a photoresponse current which is 1.5 times higher than that in the off state. ${ }^{8}$ The current ratio between light on and off states for a thin film of Te NWs and Au NPs was lower than that of the Te NW-based thin film. To investigate the photoresponse behavior of the prepared nanomaterials, we performed photovoltaic measurements of the Te NW-, Au-Te NW-, and Au-Te$\mathrm{Au} \mathrm{NW}$-based thin films. The current-voltage $(I-V)$ curves in Fig. 4A reveal the semiconductive properties of a thin film of Te NWs that have lengths and diameters of $950 \pm 160$ and $40 \pm 5$ $\mathrm{nm}$, respectively. The current increased to $c a$. $2.6 \mu \mathrm{A}$ at a biased voltage of $3 \mathrm{~V}$ when we exposed the Te NW-based thin film to light $(>725 \mathrm{~nm})$. The current that flowed when the light was on was $c a$. 13 times higher than that in the dark. The resistances of the Au-Te NW- and Au-Te-Au NW-based thin films were both lower than that of the Te NW-based thin film (Fig. 4B and C). The differential current of the Au-Te-Au NW-based thin film in 



Fig. $4 I-V$ curves of the prepared (A) Te NW-, (B) Au-Te NW-, and (C) Au-Te-Au NW-based thin films in the dark and under illumination with light (>725 nm). The insets to (A) and (B) present the time-traced currents of the Te NW- and Au-Te NW-based thin films at a bias potential of $3 \mathrm{~V}$ in the presence and absence of illumination.

the presence and absence of light was only $1 \mu \mathrm{A}$ (i.e., an increase from 86 to $87 \mu \mathrm{A}$ ) at a bias voltage of $3 \mathrm{~V}$. The insets to Fig. $4 \mathrm{~A}$ and $\mathrm{B}$ present the time-traced currents of the Te NW- and Au-Te NW-based thin films, respectively, at a bias voltage of $3 \mathrm{~V}$. We observe almost no difference in the behavior of the Au-Te-Au NW-based thin film in the presence and absence of light.

\section{Conclusion}

In summary, we have developed a green-chemical route for the growth of $\mathrm{Au} \mathrm{NPs}$ onto Te NWs. By varying the $\mathrm{pH}$, we controlled the rate of the redox reaction between $\mathrm{Au}^{3+}$ and the Te NWs and, thus, the rate of formation of the Au seeds. In the presence of $10 \mathrm{mM} \mathrm{CTAB}$, we obtained $\mathrm{Au}-\mathrm{Te}$ and $\mathrm{Au}-\mathrm{Te}-\mathrm{Au}$ NWs at $\mathrm{pH} 4.0$ and 5.0, respectively. Photovoltaic data revealed that the resistance of the Te NW-based thin films is controlled by the degree of deposition of Au NPs. We suspect that Au-Te $\mathrm{NW}$ - and/or Au-Te-Au NW-based thin films hold great potential for use in the fabrication of electronic devices.

\section{Acknowledgements}

This study was supported by the National Science Council of Taiwan under contracts 96-2627-M-002-013 and 96-2627-M-002-014.

\section{References}

1 (a) X. Peng, M. C. Schlamp, A. V. Kadavanich and A. P. Alivisatos, J. Am. Chem. Soc., 1997, 119, 7019-7029; (b) H. Gu, R. Zheng, Z. Zhang and B. Xu, J. Am. Chem. Soc., 2004, 126, 5664-5665; (c) S. C. Erwin, L. Zu, M. I. Haftel, A. L. Efros, T. A. Kennedy and D. J. Norris, Nature, 2005, 436, 91-94; (d) H. Wang, D. W. Brandl, F. Le, P. Nordlander and N. J. Halas, Nano Lett., 2006, 6, 827-832; (e) F. Vietmeyer, B. Seger and P. V. Kamat, Adv. Mater., 2007, 19, 2935-2940.

2 (a) Y.-Y. Yu, S.-S. Chang, C.-L. Lee and C. R. C. Wang, J. Phys. Chem. B, 1997, 101, 6661-6664; (b) X. Peng, L. Manna, W. Yang, J. Wickham, E. Scher, A. V. Kadavanich and A. P. Alivisatos, Nature, 2000, 404, 59-61; (c) R. Jin, Y. Cao, C. A. Mirkin, K. L. Kelly, G. C. Schatz and J. G. Zheng, Science, 2001, 294, 1901-1903; (d) Y. Sun and Y. Xia, Science, 2002, 298, 2176-2179; (e) L. Manna, D. J. Milliron, A. Meisel, E. C. Scher and A. P. Alivisatos, Nat. Mater., 2003, 2, 382-385.

3 (a) C. Pacholski, A. Kornowski and H. Weller, Angew. Chem., Int. Ed., 2004, 43, 4774-4777; (b) T. Mokari, E. Rothenberg, I. Popov, R. Costi and U. Banin, Science, 2004, 304, 1787-1790; (c) T. Mokari, C. G. Sztrum, A. Salant, E. Rabani and U. Banin, Nat. Mater., 2005, 4, 855-863; (d) A. E. Saunders, I. Popov and U. Banin, J. Phys. Chem. B, 2006, 110, 25421-25429; (e) L. Carbone, S. Kudera, C. Giannini, G. Ciccarella, R. Cingolani, P. D. Cozzoli and L. Manna, J. Mater. Chem., 2006, 16, 3952-3956.

4 D. Steiner, T. Mokari, U. Banin and O. Millo, Phys. Rev. Lett., 2005, 95, 056805.

5 A. Salant, E. A. Sadovsky and U. Banin, J. Am. Chem. Soc., 2006, 128, 10006-10007.

6 Z.-H. Lin and H.-T. Chang, Langmuir, 2008, 24, 365-367.

7 Z.-H. Lin, Z. Yang and H.-T. Chang, Cryst. Growth Des., 2008, 8, 351-357.

8 Y. Wang, Z. Tang, P. Podsiadlo, Y. Elkasabi, J. Lahann and N. A. Kotov, Adv. Mater., 2006, 18, 518-522.

9 T. Herricks, J. Chen and Y. Xia, Nano Lett., 2004, 4, 2367-2371.

10 P. H. C. Camargo, Y. Xiong, L. Ji, J. M. Zuo and Y. Xia, J. Am. Chem. Soc., 2007, 129, 15452-15453.

11 (a) B. Nikoobakht and M. A. El-Sayed, Langmuir, 2001, 17, 6368-6374; (b) J. Gao, C. M. Bender and C. J. Murphy, Langmuir, 2003, 19, 9065-9070.

12 (a) C.-C. Huang, Z. Yang and H.-T. Chang, Langmuir, 2004, 20, 6089-6092; (b) M. Grzelczak, J. Pérez-Juste, B. Rodríguez-González and L. M. Liz-Marzán, J. Mater. Chem., 2006, 16, 3946-3951; (c) M. Grzelczak, B. Rodríguez-González, J. Pérez-Juste and L. M. Liz-Marzán, Adv. Mater., 2007, 19, 2262-2266.

13 R. D. Robinson, B. Sadtler, D. O. Demchenko, C. K. Erdonmez, L. W. Wang and A. P. Alivisatos, Science, 2007, 317, 355-358. 